\title{
Emprego do carvão ativado para remoção de atrazina em água de abastecimento público
}

\section{Using activated carbon for atrazine removal from public water supply}

\author{
Edumar Ramos Cabral Coelho \\ Engenheira Civil pela Universidade Federal do Espírito Santo (UFES). Doutora em Hidráulica e Saneamento pela Escola de Engenharia de São Carlos da \\ Universidade de São Paulo (EESC/USP). Professora-associada do Departamento de Engenharia Ambiental da UFES atuando na área de Saneamentos Básico \\ e Ambiental - Vitória (ES), Brasil.

\begin{abstract}
Heverton Vazzoler
Bacharel e licenciado pleno em Química. Doutorando em Engenharia de Materiais pela Escola Politécnica da Universidade de São Paulo (POLI/USP). Professor da Coordenadoria de Engenharia de Produção do Instituto Federal do Espírito Santo - Cariacica (ES), Brasil.
\end{abstract}

\section{Waldileira Pereira Leal}

Graduada em Química Industrial pela Universidade Vale do Rio Doce. Mestranda em Engenharia Ambiental pela UFES - Vitória (ES), Brasil.

\begin{abstract}
Resumo
A atrazina é um dos herbicidas mais utilizados no mundo, sendo encontrada em águas superficiais e subterrâneas. Pertence à classe das s-triazinas, possui potencial carcinogênico e apresenta toxicidade como disruptor endócrino. A reconhecida limitação dos processos de tratamento de água, os quais empregam a coagulação química na remoção da atrazina e os efeitos na saúde, motivou este trabalho, considerando a adsorção em carvão ativado como tecnologia alternativa. O estudo experimental consistiu na caracterização física do carvão ativado e nos ensaios de adsorção em águas de diferentes conteúdos orgânicos. Foi possível verificar a capacidade do carvão ativado em remover atrazina, e o mecanismo de adsorção foi influenciado pela variação da qualidade das matrizes de água gerando isotermas com características distintas.
\end{abstract}

Palavras-chave: atrazina; carvão ativado; isotermas de adsorção; matéria orgânica natural.

\section{Abstract}

Atrazine is one of the most widely herbicides used in the world, being found in surface water and groundwater. It belongs to the s-triazines class classified as possible carcinogenic potential and toxicity presents as an endocrine disruptor. The acknowledged limitation of the water treatment processes, which employ chemical coagulation in atrazine removal and the risks to health, has motivated this work taking into regards the adsorption of activated carbon as an alternative technology. The experimental study consisted of physical characterization of activated carbon and of adsorption tests in waters of different organic content. It was possible to verify the capacity of activated carbon to remove atrazine, and the adsorption mechanism was influenced by the quality variation of water matrices generating isotherms with different characteristics.

Keywords: atrazine; activated carbon; adsorption isotherms; natural organic matter.

\section{Introdução}

A aplicação de agrotóxicos na agricultura tem gerado grandes complicações ambientais como, por exemplo, a contaminação de mananciais destinados ao abastecimento público. Quando acumulados no corpo humano, mesmo em doses relativamente pequenas, os agrotóxicos podem produzir sérios efeitos sobre a saúde. Sendo assim, a produção de água potável requer o uso de tecnologias que proporcionem diminuição de matéria orgânica e microcontaminantes para enquadramento ao padrão de potabilidade.

A atrazina (ATZ) é um dos herbicidas mais utilizados no mundo e é caracterizada por sua abrangente aplicação, alta persistência em diferentes ambientes aquáticos, capacidade de produzir efeitos nos sistemas neuroendócrino e reprodutivo, além de ser classificada como de potencial carcinogênico (FRIEDMANN, 
2002; GIBSON \& KOIFMAN, 2008). Nos Estados Unidos e na Europa, a ATZ tem sido encontrada em mananciais em concentrações variando de 2,9 a 89,0 $\mu \mathrm{g} . \mathrm{L}^{-1}$ (CEREJEIRA et al., 2003; MILTNER; BAKER; SPETH, 1989). Segundo Graymore, Stagnitti e Allinson (2001), a contaminação dos mananciais pode atingir concentrações para ATZ tão altas quanto $1.000 \mu \mathrm{g} \cdot \mathrm{L}^{-1}$ após as primeiras chuvas. No Brasil, a ATZ é utilizada nas culturas de milho, sorgo e cana-de-açúcar, e alguns estudos de monitoramento indicam sua presença em mananciais (LAABS et al., 2002; BORTOLUZZI et al., 2006). Do ponto de vista químico, a ATZ é classificada como um derivado nitrogenado levemente polar, de caráter básico. Algumas de suas propriedades incluem: pKa igual a 2,35; solubilidade em água igual a $33 \mathrm{mg} . \mathrm{L}^{-1}$ e tamanho molecular igual a $8,47 \AA$. A Figura 1 apresenta a estrutura química da ATZ.

Entre as alternativas tecnológicas para remoção da ATZ em sistemas de tratamento de água, a adsorção em carvão ativado tem sido utilizada para o enquadramento das águas de abastecimento público aos padrões de potabilidade (JIANG \& ADAMS, 2006).

A adsorção é influenciada pelas propriedades do adsorvente e do adsorvato, pela temperatura, pelo tempo de contato e pelas propriedades físico-químicas da água (GULLÓN \& FONT, 2000; JIANG \& ADAMS, 2006). As propriedades do carvão ativado relacionam-se com as estruturas porosas e os grupos químicos presentes em sua superfície. Já aquelas propriedades físicas da superfície são frequentemente descritas pela área superficial específica (ASE) e pelo tamanho dos poros, enquanto as propriedades químicas dependem da presença ou ausência de grupos ácidos ou básicos sobre sua superfície. Entre as características do



Figura 1 - Fórmula estrutural da atrazina.

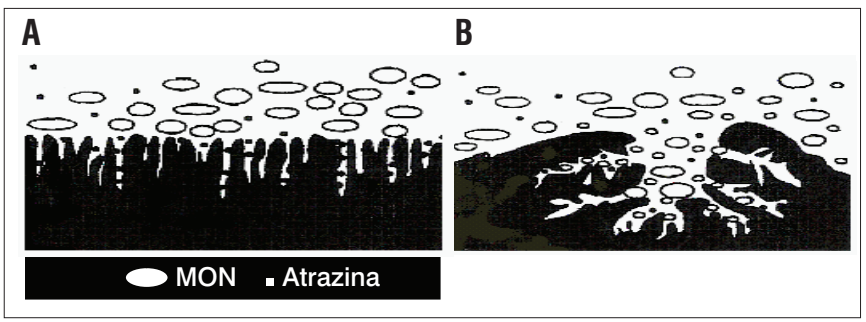

Figura 2 - Moléculas de atrazina adsorvidas em carvão ativado microporoso (A) e com variada distribuição de poros (B). Fonte: Hopman et al. (1998). adsorvato que podem influenciar o processo de adsorção estão tamanho molecular, solubilidade, pKa e natureza dos compostos substituintes dos anéis aromáticos (CASTILLA, 2004).

O carvão ativado em pó é bastante utilizado para reduzir a concentração de microcontaminantes em sistemas de tratamento de água para abastecimento público, no entanto, a presença de matéria orgânica natural (MON) pode impactar negativamente a capacidade de adsorção. A competição entre microcontaminantes e MON depende principalmente de suas características, dos pontos de vista quantitativo e qualitativo, da distribuição de poros do carvão ativado e da concentração relativa entre MON e microcontaminantes. A MON de baixo peso molecular tem sido apontada como a principal causa de competição nos poros e aquela de maior peso molecular causa bloqueio dos poros impedindo acesso do microcontaminante aos microporos (HUMBERT et al., 2008; NEWCOMBE et al., 2002).

Na Figura 2 é apresentado um esquema de adsorção das moléculas de ATZ na presença de MON em carvões de diferentes estruturas porosas. Pelekani e Snoeyink (1999) estudaram a remoção da ATZ (concentração de 45 a $305 \mu \mathrm{g}$. L-1) em águas naturais por meio de isotermas, e constataram que a distribuição do tamanho de poros é uma das mais importantes propriedades que influenciam a adsorção. Ebie et al. (2001) encontraram que a adsorção de MON ocorreu em poros de tamanhos entre 30 e $100 \AA$ na faixa dos mesoporos e que compostos orgânicos halogenados adsorveram em poros menores do que $15 \AA$.

Para avaliar a capacidade de adsorção em carvão ativado, alguns autores têm utilizado os modelos de Freundlich e Langmuir para prever a remoção de microcontaminantes, tais como microcistina, isopruton e ATZ em águas naturais como uma primeira aproximação do efeito da matéria orgânica no processo de adsorção (HUMBERT et al., 2008; LEBEAU et al., 1999; LI et al., 2002; MÜLLER; RAYA-RODRIGUEZ; CYBIS, 2009, NEWCOMBE et al., 2002; ZHANG; SHAO; KARANFIL, 2011). Os resultados encontrados limitam-se às condições experimentais devido à diversidade e variação do conteúdo orgânico nos mananciais e às características porosas do carvão ativado. Se a equação de Freundlich for empregada para descrever o equilíbrio da adsorção, pode-se observar redução da constante de adsorção ( $K f)$ com pequeno aumento do valor de $1 / n$ indicando a influência de outros adsorvatos no processo de adsorção quando comparado com a água isenta de matéria orgânica (LI et al., 2003; PELEKANI \& SNOEYINK, 2000)

Considerando os problemas de saúde pública que podem ser causados pela presença de ATZ em águas de abastecimento e a limitação dos processos de tratamento, denominados convencionais na remoção desta, a proposta deste trabalho foi avaliar a adsorção em carvão ativado por meio de isotermas em águas com diferentes conteúdos orgânicos. 


\section{Materiais e Métodos}

\section{Caracterização do carvão ativado}

O carvão utilizado foi fabricado por processo de ativação física, sendo a casca de coco utilizada como matéria-prima. As características fornecidas pelo fabricante para o carvão foram: densidade aparente de 0,45 a 0,55 g. $\mathrm{cm}^{-3}$ e índice de iodo com valor numérico próximo ao da área superficial específica, que varia de 800 a $1.000 \mathrm{~m}^{2} . \mathrm{g}$.

O índice de iodo foi determinado pelo método baseado na American Water Works Association (AWWA), citado pela norma MB 3.410 (ABNT, 1991). A distribuição de poros foi determinada pelo método BJH de Barrett, Joyner e Halenda (MARTINEZ, 1990). A ASE foi determinada construindo-se a isoterma de Brunauer, Emmett e Teller (BET).

\section{Caracterização das amostras de água para estudos de adsorção}

Os experimentos de adsorção foram realizados em diferentes amostras de águas, com a finalidade de avaliar a influência do conteúdo orgânico. As amostras foram fortificadas com ATZ na concentração de $850 \mu \mathrm{g} . \mathrm{L}^{-1}$, conduzindo a diferentes relações entre MON e ATZ. As águas utilizadas nos ensaios foram: água destilada e deionizada (ADD); bruta (AB) do Rio Jucu e filtrada (AF) do tratamento de ciclo completo abastecido pelo Rio Jucu. As amostras foram caracterizadas pela turbidez, absorbância de 254 nm, cor real, carbono orgânico total (COT), pH e ATZ. Exceto para medida de turbidez, as amostras foram filtradas em membrana de porosidade $(0,45 \mu \mathrm{m})$ para remover a matéria particulada. A turbidez foi medida em turbidímetro 2100 P da HACH Company; a absorvância e a cor verdadeira, em espectrofotômetro da Ultrospec 1000 Pharmacia Biotech; e o pH, em potenciômetro da Quaxtron modelo 8010. A medida de carbono orgânico foi obtida por meio da filtração e combustão com acidificação prévia da amostra (ácido clorídrico $2 \mathrm{~N}$ ), utilizando o equipamento TOC 5000-A-Shimadzu Corporation. Para caracterização das amostras foram utilizadas as metodologias estabelecidas pelo Standard Methods for the Examination of Water and Wastewater, $20^{\mathrm{a}}$ edição (APHA, 2000). Para cada parâmetro foram feitas análises em triplicata, e o valor final foi expresso pela média aritmética.

\section{Detecção dos resíduos de atrazina}

O padrão de ATZ adquirido foi o da Novartis, com grau de pureza de 99,9\%. A técnica empregada para sua detecção foi a espectrofotométrica na região do ultravioleta $(221,7 \mathrm{~nm})$, seguindo-se a metodologia de Baudu (2001) e a cromatográfica gasosa equipada com detector de captura de elétrons. O espectrofotômetro foi o Varian Cary 1 E, equipado com uma câmara escura termostatizada a $25^{\circ} \mathrm{C}$ e a cubeta de quartzo de caminho óptico de $10 \mathrm{~mm}$.

\section{Elaboração das isotermas de adsorção}

O método empregado para elaboração das isotermas foi o Bottle-Point, que pode ser encontrado nas seguintes referências: Baudu (2001), Pelekani e Snoeyink (1999), Randtke e Snoeyink (1983) e Wang e Alben (1998). A separação granulométrica do material foi realizada de acordo com a norma MB-3412 (ABNT, 1991a).

O carvão ativado foi lavado em ADD por 48 horas, secado em estufa a $105 \pm 5^{\circ} \mathrm{C}$ durante 24 horas e resfriado em dessecador na presença de sílica gel.

A solução padrão de ATZ foi preparada em balão volumétrico calibrado para uma concentração de $850 \mu \mathrm{g} \cdot \mathrm{L}^{-1}$, que foi estabelecido para assegurar a detecção da concentração de equilíbrio por espectrofotometria. Volumes de $250 \mathrm{~mL}$ da solução obtida foram colocados em 6 erlenmeyers em presença de diferentes massas de carvão ativado $(4,6 ; 10 ; 18 ; 25$ e $30 \mathrm{mg}) . \mathrm{O}$ pH foi ajustado para 6,0 pela adição de ácido clorídrico 0,01 Mol.L-1 e adicionou-se $5 \mathrm{~mL}$ de uma solução tampão $(\mathrm{pH}=6,0)$. Em um shaker de agitação acoplado a um banho termostatizado a $25 \pm 1^{\circ} \mathrm{C}$, a rotação foi regulada para 100 RPM e os erlenmeyers ficaram sob agitação durante um período ininterrupto de sete dias. Ao final do período do ensaio, o conteúdo dos erlenmeyers foi filtrado em membrana filtrante de 0,45 $\mu \mathrm{m}$ para posterior determinação do residual de ATZ. O mesmo procedimento foi realizado para as amostras de $\mathrm{AF}$ e $\mathrm{AB}$.

De posse dos resultados das análises de ATZ e do balanço de massa, plotaram-se as isotermas de adsorção para os diferentes tipos de águas, com a massa adsorvida de ATZ no eixo das ordenadas (mg. $\mathrm{g}^{-1}$ ) e a concentração de ATZ residual de equilíbrio no eixo das abscissas (mg. $\left.\mathrm{L}^{-1}\right)$.

As isotermas derivadas teoricamente, como a de Langmuir, ou empiricamente, como a de Freundlich, são frequentemente representadas por equações matemáticas que relacionam diretamente a massa adsorvida e a concentração do adsorvato. Os modelos consistem na utilização de regressão linear aplicada à Equação 1 para o Modelo de Langmuir e à 2 para o de Freundlich. A partir de tais equações foi possível obter os valores das constantes de capacidade de adsorção e de afinidade de adsorção para o contaminante no carvão ativado em estudo.

$[Q e]=\frac{K_{L} C_{m} C e}{1+K_{L} C e}$
$Q_{e}=K_{f} C e^{\frac{1}{n}}$ 
Onde,

$\mathrm{K}_{\mathrm{L}}$ é a constante de Langmuir;

$\mathrm{K}_{\mathrm{f}}$ é a constante de capacidade de adsorção segundo modelo de Freundlich:

$1 / n$ é a constante de afinidade do adsorvato,

$C_{e}$ é a concentração da fase fluida no equilíbrio (mg. $\left.\mathrm{L}^{-1}\right)$,

$\mathrm{C}_{\mathrm{m}}$ é a capacidade máxima de adsorção em mg. $\mathrm{g}^{-1}$, segundo modelo de Langmuir, e que representa a massa adsorvida por massa de adsorvente (mg.g $\left.{ }^{-1}\right)$.

A isoterma de Freundlich é a mais utilizada a fim de descrever os processos de adsorção para água ao abastecimento público devido a sua proximidade de correlação com os dados experimentais.

\section{Resultados e discussão}

Os parâmetros utilizados na caracterização das diferentes águas de estudo estão apresentados na Tabela 1 .

\section{Caracterização do carvão ativado}

\section{Área superficial específica e distribuição de porosidade}

A ASE e a distribuição de porosidade são índices físicos utilizadas para avaliar a capacidade de adsorção, devendo os valores da ASE estarem próximos ao índice de iodo. Com o traçado da isoterma BET, pode-se observar que a maior parte da estrutura do carvão é constituída por microporos. Os resultados estão dispostos na Tabela 2.

Tabela 1 - Parâmetros de qualidade dos tipos de água estudados.

\begin{tabular}{lccc} 
Parâmetros & $\begin{array}{c}\text { Águas destilada } \\
\text { e deionizada }\end{array}$ & $\begin{array}{c}\text { Água } \\
\text { filtrada }\end{array}$ & $\begin{array}{c}\text { Água } \\
\text { bruta }\end{array}$ \\
\hline $\mathrm{pH}$ & $6,8 \pm 0,0$ & $6,0 \pm 0,1$ & $7,0 \pm 0,1$ \\
\hline Turbidez (UT) & $\leq 0,5$ & $1,2 \pm 0,1$ & $92,0 \pm 1,0$ \\
\hline Cor real (mg.L-1 Pt-Co) & $\leq 2,0$ & $8,0 \pm 0,1$ & $36,0 \pm 1,0$ \\
\hline Absorbância de $254 \mathrm{~nm}$ & - & $0,012 \pm 0,001$ & $0,044 \pm 0,001$ \\
\hline COT (mg.L-1) & $\leq 0,30$ & $1,20 \pm 0,10$ & $3,80 \pm 0,10$ \\
\hline COT/ATZ & - & 1,4 & 4,5 \\
\hline ABS/ATZ & - & 1,4 & 5,2 \\
\hline
\end{tabular}

COT: carbono orgânico total; ATZ: atrazina; ABS: absorbância.

Tabela 2 - Resultados da análise textural do carvão ativado.

\begin{tabular}{lc} 
Análises & Valores \\
\hline Área superficial específica BET $\left(\mathrm{m}^{2} \cdot \mathrm{g}^{-1}\right)$ & 567 \\
Volume dos microporos $\left(\mathrm{cm}^{3} \cdot \mathrm{g}^{-1}\right)$ & 0,315 \\
Volume dos mesoporos $\left(\mathrm{cm}^{3} \cdot \mathrm{g}^{-1}\right)$ & 0,072
\end{tabular}

BET: Brunauer, Emmett e Teller.
Em relação à área superficial específica, pode-se notar que o carvão ativado tem um valor próximo àquele utilizado por Jiang e Adams (1996) para remoção de ATZ igual a $546 \mathrm{~m}^{2} \cdot \mathrm{g}^{-1}$. Na análise da distribuição de porosidade foi possível detectar microporo mínimo de $17,8 \AA$, sendo este enquadrado pela International Union of Pure and Applied Chemistry (IUPAC) na classificação de microporo secundário (8 a 20 Å). A molécula de ATZ tem dimensão próxima a $8,47 \AA$, havendo a condição física de penetrar em tais microporos e ser adsorvida.

\section{Número de iodo}

$\mathrm{Na}$ análise do índice de iodo, obteve-se um resultado de $530 \mathrm{mg} \cdot \mathrm{g}^{-1}$ que se enquadra nas especificações da AWWA, ou seja, $500 \mathrm{mg} \cdot \mathrm{g}^{-1}$ para aplicação em tratamento de água. A NBR 12073 da Associação Brasileira de Normas Técnicas (ABNT) preconiza um valor mínimo de $600 \mathrm{mg} \cdot \mathrm{g}^{-1}$.

\section{Ensaios de adsorção}

Os ensaios de adsorção foram realizados com adição de ATZ em concentração de $850 \mu \mathrm{g} . \mathrm{L}^{-1}$, tempo de contato de sete dias, $\mathrm{pH}$ ajustado para seis e temperatura de $25 \pm 1^{\circ} \mathrm{C}$. Foram traçadas isotermas em três matrizes de água: destilada e deionizada, filtrada natural e bruta do Rio Jucu, provenientes de uma estação de tratamento de água (ETA) do tipo convencional. As características das águas foram apresentadas na Tabela 1. As isotermas de adsorção estão representadas nas Figuras 3 a 5.

A configuração da isoterma é o primeiro indicativo para explicar o fenômeno da adsorção. Carvões ativados que possuem grande capacidade adsortiva têm isotermas mais côncavas, indicando que grandes quantidades de massa de ATZ podem ser adsorvidas por unidade de massa de carvão ativado (CASTILLA, 2004). A isoterma em água destilada possui uma configuração característica dos adsorventes microporosos, os quais têm o tamanho dos poros pouco maior que o diâmetro molecular do adsorvato (MARTINEZ, 1988). Sobre os perfis, o primeiro fato que chama atenção é a gradativa perda da concavidade no formato das

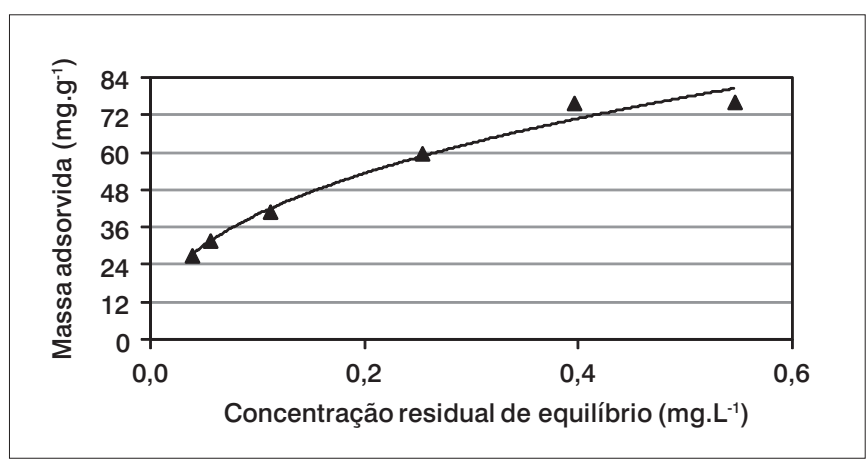

Figura 3 - Isoterma de adsorção elaborada em água destilada e deionizada, $\mathrm{pH}=6,0 ; \mathrm{T}=25 \pm 1^{\circ} \mathrm{C}$. 
isotermas de adsorção realizadas em águas bruta e filtrada em relação à isoterma realizada em água destilada.

A partir das formas linearizadas das isotermas de Langmuir e de Freundlich, foi possível determinar os parâmetros de adsorção que se encontram apresentados na Tabela 3.

O modelo de Freundlich foi utilizado para discussão dos resultados, considerando que o mesmo apresentou maiores valores do coeficiente de determinação. O parâmetro $1 / n$ mostrou valores inferiores a 1 , indicando que a adsorção de ATZ em tal carvão ativado é considerada favorável. O parâmetro $\mathrm{K}_{\mathrm{f}}$ em ADD $(104,71)$ foi inferior ao encontrado por Adams e Watson (1996) de 467,00 entre os limites de 24,4 a 467,0 referenciados por Adams (2003) em águas destilada e deionizada para remoção de ATZ, e foi superior ao encontrado por Lebeau et al. (1999), 88,7 [(mg. $\mathrm{g}^{-1}$. ( $\left.\left.\mathrm{L} \cdot \mathrm{mg}^{-1}\right)^{1 / \mathrm{n}}\right]$. Miltner et al. (1989) encontraram valor de $\mathrm{K}_{\mathrm{f}}$ em ADD igual a 283,0, e os de Li et al. (2003) foram de 121,0 e 138,0 [(mg.g $\left.{ }^{-1} \cdot\left(\mathrm{L}^{\mathrm{m}} \mathrm{mg}^{-1}\right)^{1 / \mathrm{n}}\right]$ em dois carvões distintos.

No entanto, as características para distribuição de porosidade, de ASE, dos teores de cinzas e material volátil e de química superficial influenciaram a adsorção de ATZ no carvão ativado empregado neste trabalho. Em relação às características da ATZ que influenciaram a adsorção, pode-se citar sua moderada polaridade e baixa solubilidade que determinam a interação hidrofóbica com o carvão ativado. Talvez este seja o maior desafio em se prever a remoção de contaminantes por adsorção em carvão ativado, ou seja, a definição de quais características físicas e químicas do

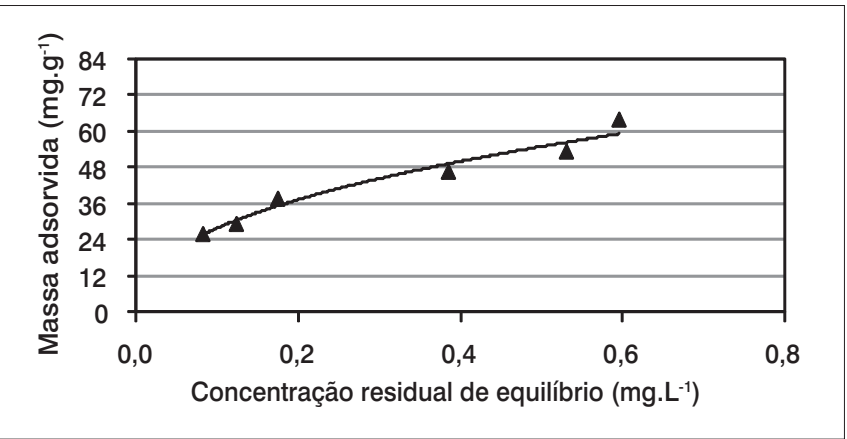

Figura 4 - Isoterma de adsorção elaborada em água efluente do filtro, $\mathrm{pH}=6,0$ e $\mathrm{T}=25 \pm 1^{\circ} \mathrm{C}$. carvão ativado são mais importantes para que este material seja utilizado na remoção de microcontaminantes em sistemas de tratamento de água.

O modelo de Freundlich indicou um bom ajuste, tanto para água destilada como para filtrada da ETA. Com relação à avaliação das isotermas em águas filtrada e bruta, pode-se notar pequena variação no valor da constante $1 / n$ e diminuição no valor de $\mathrm{K}_{\mathrm{f}}$ que foi sensível à variação da qualidade das águas. Redução no valor de $\mathrm{K}_{\mathrm{f}}$ pode ser interpretada em termos de competição e bloqueio de poros, e o valor de $n$ pode ser interpretado como variação em termos da energia de competição (PELEKANI \& SNOEYINK, 1999). Segundo Li et al. (2003), se as isotermas em presença de matéria orgânica são paralelas àquelas traçadas em água destilada, há uma pequena interferência pelo bloqueio de poros no processo de adsorção.

Li et al. (2003) estudaram a competição entre ATZ (215 Dalton) e poliestireno sulfonado (1800 Dalton) e ATZ e 1,4 diclorobenzeno (147 Dalton) com o intuito de avaliar a interferência de matéria orgânica com pesos moleculares diferentes na adsorção de ATZ, utilizando isotermas de Freundlich em carvões com diferentes volumes de mesoporos. Um dos carvões utilizados possuía volume de mesoporo igual ao do presente trabalho, portanto serão somente discutidos os resultados referentes a este carvão. No ensaio que avaliou a competição entre poliestireno sulfonado e ATZ, a redução na capacidade adsortiva para a ATZ foi pequena e atribuída à competição nos mesoporos. Quando

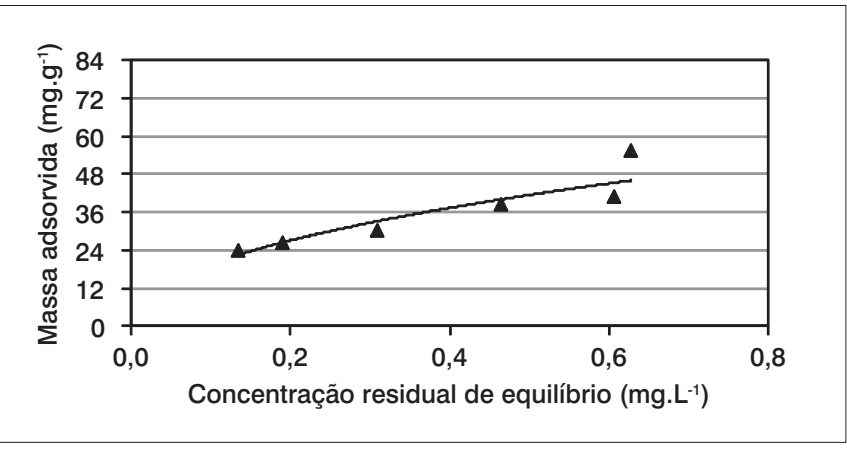

Figura 5 - Isoterma de adsorção elaborada para água bruta do Rio Jucu, $\mathrm{pH}=6,0$ e $\mathrm{T}=25 \pm 1^{\circ} \mathrm{C}$.

Tabela 3 - Coeficientes experimentais de ajuste linear das isotermas de Langmuir e Freundlich para atrazina.

\begin{tabular}{|c|c|c|c|c|c|}
\hline \multirow{2}{*}{ Tipo de água } & \multicolumn{2}{|c|}{ Isoterma de Langmuir } & \multicolumn{3}{|c|}{ Isoterma de Freundlich } \\
\hline & b & $\mathbf{R}^{2}$ & $K_{f}$ & $1 / n$ & $\mathrm{R}^{2}$ \\
\hline Água destilada e deionizada (ADD) & 80,65 & 0,9681 & 104,71 & 0,41 & 0,9909 \\
\hline Água efluente do filtro (AF) & 68,49 & 0,9575 & 74,13 & 0,42 & 0,9704 \\
\hline Água bruta do Rio Jucu (AB) & 56,82 & 0,8698 & 57,54 & 0,46 & 0,8769 \\
\hline
\end{tabular}

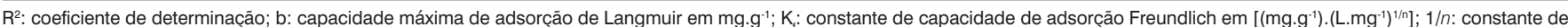
heterogeneidade e afinidade de Freundlich. 
foi avaliada a competição de ATZ e 1,4 diclorobenzeno ocorreu significativa redução na capacidade de adsorção, 74\%, devido à competição dos compostos nos microporos. Como as isotermas foram paralelas, o efeito de bloqueio dos poros foi considerando insignificante. Pelekani e Snoeyink (1999) estudaram a remoção do pesticida alacloro em carvão ativado e encontraram um decréscimo de $31 \%$ na constante $\mathrm{K}_{\mathrm{f}}$ para águas enriquecidas com ácidos húmicos. No entanto, Speth e Miltner (1990) observaram decréscimo de $35 \%$ para os valores da constante $\mathrm{K}_{\mathrm{f}}$ obtidos em águas naturais quando comparados àqueles encontrados em águas destilada e deionizada para a adsorção da ATZ. No presente trabalho, na água efluente do filtro da ETA, o valor de $\mathrm{K}_{\mathrm{f}}$ sofreu redução de 29\%, indicando interferência de outros adsorvatos para a relação COT/ATZ e ABS/ATZ igual a 1,4. Os resultados evidenciaram perda na capacidade adsortiva, que pode ocorrer pelo fenômeno de competição dos compostos da água natural com as moléculas de ATZ, uma vez que as isotermas são paralelas, ou seja, possuem valores de $1 / n$ muito próximos.

Para a $A B$, o aumento da constante $1 / n$ e a diminuição do valor de $\mathrm{K}_{\mathrm{f}}$ em $50 \%$ também indicaram influência de outros adsorvatos na adsorção da ATZ, e o modelo de Freundlich não apresentou bom ajuste para descrever o fenômeno neste tipo de água. É interessante também observar que as relações COT/ATZ igual a 4,5 e ABS/ATZ igual a 5,2 são bem superiores que as relações em AF, o que mostra a importância da relação entre as concentrações da MON e do microcontaminante que se deseja remover. Outros modelos matemáticos podem ser usados para descrever os efeitos de competição no processo de adsorção, tais como: a teoria de IAST (the ideal adsorbed solution theory) e as simplificações. Esses modelos também apresentam limitações, sendo a principal descrita por Ebie et al. (2001) e Newcombe et al. (2002), por não levar em conta o bloqueio dos poros como um mecanismo potencial de competição. Li et al. (2003), de posse dos valores dos parâmetros da isoterma de Freundlich para ATZ e p-DCB, utilizaram o modelo IAST com o objetivo de descrever a adsorção competitiva em sistema bicomponente, encontrando menor capacidade do que os obtidos pelos dados experimentais. A explicação dos autores é a possível adsorção do $p$-DCB em poros de tamanho menores não acessíveis às moléculas de ATZ, e o modelo IAST é baseado na premissa que todos os adsorvatos têm a mesma acessibilidade aos poros.

Apesar da constatação do efeito das diferentes frações de peso molecular da MON na remoção da ATZ, existe limitação na separação das diferentes frações para avaliar a interferência na capacidade adsortiva do carvão em águas naturais. Sendo assim, as isotermas são importantes como indicação da aplicação de determinado carvão ativado na remoção de contaminantes em águas naturais que apresentam grande variabilidade na distribuição de peso molecular da MON presente.

Para ampliar a aplicação do carvão ativado em águas naturais, estudos têm sido realizados com carvões de diferente distribuição de porosidade e com aqueles denominados supercarvão ativado ou carvão nanotubos (ANDO et al., 2010; LI et al., 2003; ZHANG; SHAO; KARANFIL, 2011).

A comparação da capacidade de adsorção dos carvões ativados em águas naturais em diferentes trabalhos é tarefa difícil devido às variadas condições de estudo, tais como: concentrações de ATZ, valores de $\mathrm{pH}$, características físicas e químicas do carvão ativado e variabilidade das características da MON. Pode-se perceber também que a relação COT/ATZ indicou interferência significativa no processo de adsorção, diminuindo o equilíbrio de adsorção quando tal relação aumentou.

\section{Conclusões}

Com o aumento das evidências dos potenciais efeitos da ATZ como disruptor endócrino, sua remoção em sistemas de tratamento de água para abastecimento público torna-se relevante. Devido ao fato de considerar o carvão ativado como tecnologia para remoção da ATZ, o presente trabalho foi realizado. Ainda, dentro das condições estudadas foi possível verificar as seguintes considerações finais:

- a análise física da distribuição de porosidade evidenciou um carvão ativado de predominância microporosa com tamanho mínimo de poro detectável igual a 17,8 A, que é aproximadamente o dobro da dimensão da molécula de ATZ, indicando a possibilidade de sua utilização como material adsorvedor;

- a regressão linear para o modelo de adsorção de Freundlich mostrou melhor ajuste quando comparada ao de Langmuir para os diferentes tipos de água. Para isoterma de Freundlich, os valores de $1 / n$ foram menores que a unidade, mostrando ser favorável o processo de adsorção da ATZ no carvão estudado. Os valores de $\mathrm{K}_{\mathrm{f}}$ encontrados para água destilada estavam dentro dos limites apresentados pela literatura, confirmando a capacidade do carvão ativado estudado em remover ATZ;

- nos ensaios de adsorção, pode-se verificar a adsorção competitiva entre a ATZ e a matéria orgânica, indicada pelo aumento dos valores de $1 / n$ e a diminuição dos de $\mathrm{K}_{\mathrm{f}}$ à medida que a relação COT/ATZ aumentava;

- o modelo de Freundlich apresentou bom ajuste em água filtrada para relação de COT/ATZ e ABS/ATZ igual a 1,4, o que não ocorreu para água bruta às relações COT/ATZ igual a 4,5 e ABS/ATZ igual a 5,2. 


\section{Referências}

ADAMS, C.D. \& JIAN, G.H. (2006) Treatability of s-triazine by conventional drinking water treatment technologies. Water Research, v. 40, p. 1657-1667.

ADAMS, C.D. \& WATSON, T.L. (1996) Treatability of s-triazine herbicide metabolites using powered activated carbon. Journal of Environmental Engineering, v. 122, n. 4, p. 327-330.

ANDO, N.; MATSUI, Y.; KUROTOBI, R.; NAKANO, Y.; MATSUSHITA, T.; OHNO, K. (2010) Comparison of natural organic matter adsorption capacities of super-powered activated carbon and powered activated carbon. Water Research, v. 44, p. 1227-4133.

AMERICAN PUBLIC HEALTH ASSOCIATION - APHA. (2000) Standard Methods for the Examination of Water and Wastewater. $20^{\text {th }}$ edition. New York: APHA, AWWA, WPCF.

ASSOCIAÇÃO BRASILEIRA DE NORMAS TÉCNICAS (ABNT). (1991a) Carvão ativado pulverizado - Determinação granulométrica - Método de Ensaio - MB-3412. Rio de Janeiro.

ASSOCIAÇÃO BRASILEIRA DE NORMAS TÉCNICAS (ABNT). (1991b) Carvão ativado pulverizado para tratamento de água - Especificação EB-2133. Rio de Janeiro.

ASSOCIAÇÃO BRASILEIRA DE NORMAS TÉCNICAS (ABNT). (1991C) Carvão ativado pulverizado - Determinação do número de iodo - MB 3410. Rio de Janeiro.

BAUDU, J.M. (2001) Prevision de l'adsorption de molecules organiques en solution aqueuse en fonctions de quelques caracteristiques physico-chimiques de charbons actifs. Water Quality Research, v. 36, n. 4 , p. 631-657.

BORTOLUZZI, E.C.; RHEINHEIMER, D.S.; GONÇALVES, C.S.; PELLEGRINI, J.B.R.; ZANELLA, R.; COPETTI, A.C.C. (2006) Contaminação de águas superficiais por agrotóxicos em função do uso do solo numa microbacia hidrográfica de Agudo, RS. Revista Brasileira de engenharia agrícola ambiental, v. 10, n. 4, p. 881-887.

CASTILLA, C.M. (2004) Adsorption of organic molecules from aqueous solutions on carbon materials. Carbon, v. 42, p. 83-94.

CEREJEIRA, M.J.; VIANA, P.; BATISTA, S.; PEREIRA, T.; SILVA, E.; VALERIO, M.J.; SILVA, A.; FERREIRA, M.; FERNANDES, A.M. (2003) Pesticides in Portuguese Surface and Ground Waters. Water Research, v. 37, p. $1055-1063$

EBIE, K.; LI, F.; AZUMA, Y.; YUASA, A.; HAGISHITA, T. (2001) Pore distribution effect of activated carbon in adsorbing organic micropollutants from natural water. Water Research, v. 35, n. 1, p. 167-179.

FRIEDMANN, A.S. (2002) Atrazine inhibition of testosterone production in rat males following peripuberal exposure. Reproductive Toxicology, v. 16, p. 275-279.

GIBSON, G. \& KOIFMAN, S. (2008) Consumo de agrotóxicos e distribuição temporal da proporção de nascimentos masculinos no
Estado do Paraná, Brasil. Revista Panamericana de Saúde Pública, v. 24, n. 4 , p. 240-247.

GRAYMORE, M.; STAGNITTI, F.; ALLINSON, G. (2001) Impacts of atrazine in aquatic ecosystems. Environment International, v. 26, n. 7-9, p. 483-495.

GULLÓN, M. \& FONT, R. (2000) Dynamic Pesticide Removal with Activated Carbon Fibers. Water Research, v. 35, n. 2, p. 516-520.

HOPMAN, R.; VAN DER HOEK, J.P.; VAN PAASSEN, J.M. (1998) Impact of natural organic matter (NOM) on drinking-water treatment. Water Supply, v. 16, p. 497-509.

HUMBERT, H.; GALLARD. H.; SUTY, H.; CROUÉ, J. (2008) Natural organic matter (NOM) and pesticides removal using a combination of ion exchange resin and powdered activate carbon (PAC). Water Research, v. 42, p. $1635-1643$.

JIANG, H. \& ADAMS, C. (2006) Treatability of chloro-s-triazines by conventional drinking water treatment technologies, Water Research, v. 40, p. $1657-1667$.

LAABS, V.; AMELUNG, W.; PINTO, A.A.; WANTZEN, M.; DA SILVA, C.J.; ZECH, W. (2002) Pesticides in Surface Water, Sediment, and Rainfall of the Northeastern Pantanal Basin, Brazil. Journal of Environmental Quality, v. 31, p. $1636-1648$

LEBEAU, T.; LELIĖVRE, D.; WOLBERT, D.; LAPLANCHE, A.; PRADOS, M.; CÔTÉ, P. (1999) Effect of natural organic matter loading on the atrazine adsorption capacity of an aging powdered activated carbon slurry. Water Research, v. 33, p. 1695-1705.

LI, Q.; SNOEYINK, V.L.; CAMPOS, C.; MARIÑAS, B.J. (2003) Elucidating competitive adsorption mechanisms of atrazina and NOM using model compounds. Water Research, v. 37, p. 773-784.

LI, Q.; SNOEYINK, V.L.; CAMPOS, C.; MARIÑAS, B.J. (2002) Displacement Effect of NOM on Atrazine Adsorption by PACs with different pore size distributions. Environmental Science \& Technology, v. 36, p. $1510-1515$

MARTINEZ, J.M.M. (1990) Adsorción física de gases y vapores por carbones. Universidade de Alicante, Alicante, Espanha, Editora Secretariado de Publicaciones de La Universidad de Alicante, 376p.

MILTNER, R.J.; BAKER, D.B.; SPETH, T.F. (1989) Treatment of seasonal pesticides in surface water. Journal of American Water Woorks Association, v. 81, p. $43-52$.

MÜLLER, C.C.; RAYA-RODRIGUEZ, M.T.; CYBIS, L.F. (2009) Adsorção em carvão ativado em pó para remoção de microcistina de água de abastecimento público. Revista de Engenharia Sanitária e Ambiental, v. 14, n.1, p. 29-38.

NEWCOMBE, G.; MORRISON, J.; HEPPLEWHITE, C.; KNAPPE, D.R.U. (2002) Simultaneous adsorption of MIB and NOM onto activated carbon: II. Competitive effects. Carbon, v. 40, p. 2147-2156. 
PELEKANI, C. \& SNOEYINK, V. (2000) Competitive adsorption between atrazine and Metilene blue on activated carbon: the importance of pore size distribution. Carbon, v. 38, n. 10, p. 1423-1436.

RANDTKE, S.J. \& SNOEYINK, V.L. (1983) Evaluating GAC adsorptive capacity. Journal of American Water Works Association, v. 75, n. 8, p. 406-413.
SPETH, T.F. \& MILTNER, R.J. (1990) Technical Note: Adsorption Capacity of GAC for Synthetic Organics. Journal of American Water Works Associtation, v. 82, n. 2, p. 72-75.

ZHANG, S.; SHAO, T.; KARANFIL, T. (2011) The effects of dissolved natural organic matter on the adsorption of synthetic organic chemicals by activated carbons and carbon nanotubes. Water Research, v. 45, p. 1378-1386. 